\title{
CENÁRIOS AMBIENTAIS PARA O ORDENAMENTO TERRITORIAL DE ÁREAS DE PRESERVAÇÃO PERMANENTE NO MUNICÍPIO DE SOROCABA, SP ${ }^{1}$
}

\author{
Kaline de Mello², Laís Petri ${ }^{3}$ Eliana Cardoso Leite ${ }^{4}$ e Rogério Hartung Toppa ${ }^{4}$
}

\begin{abstract}
RESUMO - Este estudo objetivou analisar a importância das Áreas de Preservação Permanente (APP) para a manutenção dos remanescentes florestais no município de Sorocaba, SP. O mapa desses remanescentes foi elaborado com base em fotografias aéreas de 2006, e mapas de hidrografia e de declividade foram usados para determinação das APP. Compararam-se dois cenários: real (mata atual) e ideal (mata atual acrescida da restauração das APP), com base em métricas da paisagem. Os resultados indicaram que 19\% do município se enquadra como APP e, dessa área, apenas $45 \%$ possui cobertura florestal. As APP preservadas representam 50\% do total de cobertura florestal da paisagem. A restauração das APP representaria aumento de 16,68\% para 28\% de cobertura florestal da paisagem e possibilitaria o surgimento de fragmentos maiores que 3.000 ha, enquanto no cenário real os maiores fragmentos atingem 300 ha. Os remanescentes florestais se apresentaram intimamente ligados às APP: margens de rios, áreas alagáveis e encostas, onde a ocupação urbana foi dificultada e existe o instrumento legal de proteção. Esse quadro evidencia que as APP possuem papel fundamental na conservação florestal em um município bastante urbanizado, onde as áreas naturais sofrem muita pressão. A avaliação dos cenários mostrou que a incorporação dos dispositivos legais no planejamento e ordenamento territorial subsidia as estratégias voltadas para a manutenção de hábitats naturais e, consequentemente, a diminuição dos efeitos negativos sobre a biodiversidade. Entretanto, as mudanças recentes no Código Florestal podem representar um retrocesso no cenário ambiental nacional diante da crescente urbanização.
\end{abstract}

Palavras-chave: Paisagens fragmentadas; Política ambiental; Sistema de Informações Geográficas (SIG).

\section{ENVIRONMENTAL SCENARIOS FOR LAND PLANNING OF PERMANENT PRESERVATION AREAS IN SOROCABA, SP}

\begin{abstract}
The present study aimed to analyze the importance of the Permanent Preservation Areas (PPA) for the forest remnants maintenance in the city of Sorocaba, State of São Paulo, Brazil. A forest remnants map was made using aerial photographs from 2006, and hydrographic and slope maps were used to PPA determination. Two scenarios were compared with landscape metrics: real (with current forest) and ideal (current forest adding the PPA restoration). The results showed that 19\% of the city territory were classified as PPA, and from this area, only 45\% have forest cover. The preserved PPA represents $50 \%$ of all forest cover in the landscape. Restoring the PPA would increase from $16.68 \%$ to $28 \%$ of forest cover landscape and enable the emergence of fragments larger than 3,000 ha. In the real scenario, the bigger fragments have $300 \mathrm{ha}$. The presence of forest remnants is closely related to PPA areas: riverbanks, floodplains and hillside, where urban occupation was hampered and there is legal protection. The situation shows that the PPA play a fundamental role in forest patches conservation in a largely urbanized city, where natural areas support pressure. The evaluation of the scenarios showed that the incorporation of the legal provisions in planning and territorial ordering subsides the strategies for the maintenance of natural habitats and, consequently the reduction of the negative effects on biodiversity. However, the recent changes in Forest Code may represent a retrocession in the environmental scenario against to the increasing urbanization.
\end{abstract}

Keywords: Fragmented landscapes; Environmental policy; Geographic Information System (GIS).

\footnotetext{
${ }^{1}$ Recebido em 07.12.2012 aceito para publicação em 11.03.2014.

${ }^{2}$ Programa de Pós-Graduação em Engenharia de Sistemas Agrícolas, Universidade de São Paulo, Escola Superior de Agricultura “Luiz de Queiroz”. E-mail: <kaline.mello@gmail.com>.

${ }^{3}$ Florestal Atlântica Comércio e Serviços Ambientais LTDA. E-mail: <laispetri@gmail.com>.

${ }^{4}$ Departamento de Ciências Ambientais, Universidade Federal de São Carlos, Sorocaba, SP. E-mail: <eliana.leite@ufscar.br>e <toppa@ufscar.br>.
} 


\section{INTRODUÇÃO}

Atualmente, 68,7\% da população mundial reside em áreas urbanas (UN, 2010), e no Brasil essa porcentagem chega a 80\% (IBGE, 2010a). A urbanização possui como impacto direto a transformação do ambiente, alterando a ocupação dos solos e gerando um padrão espacial complexo de manchas de vegetação natural cada vez mais isoladas e menores inseridas em uma matriz inóspita para muitas espécies (GRIMM et al., 2000; PICKETT et al., 2001; KAYE et al., 2006; ALBERTI, 2010). O processo contínuo de urbanização gera paisagens cada vez mais homogêneas com alto grau de fragmentação de hábitat, causando a perda de biodiversidade, dos processos e das funções e dinâmicas ecológicas e, consequentemente, diminuição da disponibilização dos serviços ecossistêmicos (GRIMM et al., 2008; ALBERTI, 2010; STEINER, 2011).

A sustentabilidade do ambiente urbano depende da manutenção dos processos ecológicos, que podem ser potencializados pela presença de elementos na paisagem que aumentem a conectividade funcional entre os remanescentes maiores de vegetação natural, como corredores ou trampolins ecológicos (CHETKIEWICZ et al., 2006; LUSSIER et al., 2006; CASTÉLON; SIEVING, 2007; ALBERTI, 2010; ZHOU et al., 2011), e esse pode ser o papel das Áreas de Preservação Permanente (APP) (METZGER, 2010). As APP, além de protegerem áreas fisicamente sensíveis e contribuírem para a manutenção da qualidade da água, exercem grande importância para a permanência e deslocamento da fauna e flora local. Estudos evidenciam a importância da manutenção de vegetação e árvores nativas na paisagem urbana para a contenção da perda da riqueza de espécies (MCKINNEY, 2002), como de espécies de aves (OLIVER et al., 2011; REIS et al., 2012), mamíferos carnívoros (BATEMAN; FLEMING, 2012), pequenos mamíferos (GOMES et al., 2011), morcegos insetívoros e a biomassa de insetos (THRELFALL et al., 2012).

Análises qualitativas e quantitativas do processo de fragmentação da vegetação nativa em matriz urbana têm sido pouco estudadas, apesar de fundamentais para que se tenham argumentos e direcionamentos nas tomadas de decisão pelos gestores das paisagens urbanas (SCOLOZZI; GENELETTI, 2012). A análise da paisagem utilizando técnicas de Sistema de Informações Geográficas (SIG) e métricas da paisagem auxilia na espacialização das informações sobre mudanças do uso do solo e tipo de cobertura, possibilitando a criação de cenários atuais e perspectivas futuras robustas do ambiente (GRIMM et al., 2000, 2008; YORK et al., 2011). As métricas da paisagem são reconhecidas atualmente como bons indicadores de biodiversidade e são usadas em diferentes etapas do planejamento em conservação (LINDENMAYER et al., 2008; BANKS-LEITE et al., 2011; SCHINDLER et al., 2012).

Nesse sentido, este trabalho teve como principais objetivos mapear e quantificar as Áreas de Preservação Permanente e analisar o uso do solo nessas áreas, além de comparar o cenário atual com um possível cenário ideal, ou seja, onde a legislação seria cumprida, a fim de subsidiar as estratégias de conservação e restauração na área estudada.

\section{MATERIAL E MÉTODOS}

\section{1. Área de estudo}

O município de Sorocaba localiza-se no Estado de São Paulo, entre as coordenadas UTM: $236243-$ 265122 m E, 7388590 - 7415800 m N (Figura 1). Com apenas $17,5 \%$ de Zona Rural, a paisagem atual de Sorocaba encontra-se bastante urbanizada, e sua vegetação original de transição entre os biomas de Mata Atlântica e Cerrado (KRONKA, 2005) possui alto grau de fragmentação. Segundo a classificação de Koeppen, Sorocaba encontra-se em uma faixa de transição entre o clima tipo Cfa e Cwa e localiza-se na zona de transição entre o Planalto Atlântico e a Depressão Periférica. O relevo é composto por Colinas Médias, Morretes Alongados Paralelos, Morretes Alongados e Espigões, com amplitudes locais inferiores a $100 \mathrm{~m}$ (PONÇANO et al., 1981).

\subsection{Metodologia}

O mapeamento dos fragmentos de vegetação natural foi realizado no programa MapInfo 9.0 pelo método de classificação digital visual, por meio de vetorização em tela, escala 1:2.000, com base em fotografias aéreas do ano 2006, com resolução de 0,4 m.

Para obter a concordância entre a verdade terrestre e o mapa de fragmentos de vegetação natural produzido, foi estimada a exatidão do mapa por meio de matriz de erro e coeficiente Kappa (CONGALTON; GREEN, 1998). Para tanto, foram determinados 200 pontos na imagem para verificação em campo do uso do solo, 


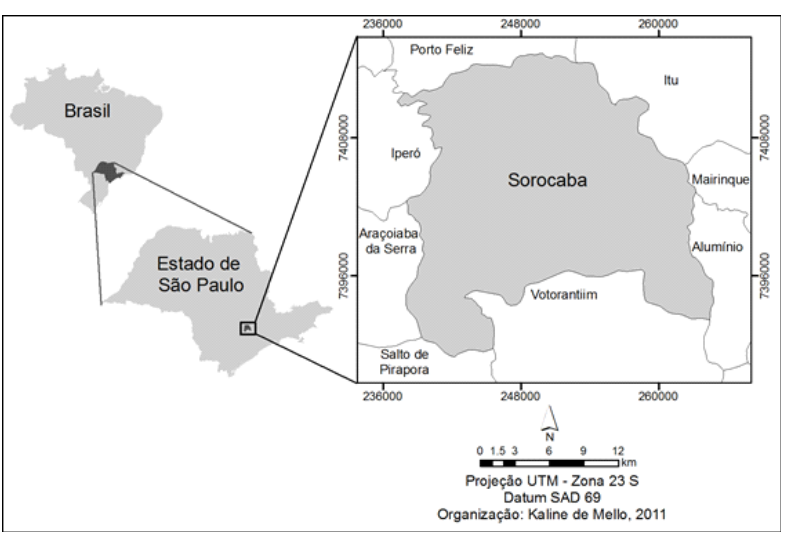

Figura 1 - Localização do município de Sorocaba, Estado de São Paulo, Brasil.

Figure 1 - Location of the city of Sorocaba, São Paulo State, Brazil.

comparando-se a classe adotada no mapa e a classe real a que pertence o ponto em campo, permitindo a determinação de erros de omissão (omissão no mapa de determinada feição constatada em campo) e erros de comissão (atribuição no mapa de determinada feição a uma classe a qual ela não pertence). $\mathrm{O}$ valor do índice Kappa indica a qualidade da classificação, variando de 0 a 1 , e, quanto mais se aproxima de 1 , mais a classificação se aproxima da realidade (MOREIRA, 2001). O índice Kappa obtido neste trabalho foi de 0,79, indicando que o resultado pode ser considerado excelente, segundo a classificação de Rosner (2006), que avalia como excelente valor de Kappa maior que 0,75 . Os erros de comissão se deram principalmente devido à confusão com alguns estágios de crescimento de Eucalyptus sp., tendo sido encontrado apenas um erro de omissão.

A escala refinada que foi utilizada neste trabalho (1:2.000) permitiu o mapeamento de pequenos fragmentos (< 1 ha). Ribeiro et al. (2009), trabalhando com escala de 1:50.000, identificaram erros do mapa em relação à dificuldade do mapeamento correto de fragmentos menores que 30 ha; matas de encosta, muitas vezes confundidas com plantação de Eucalyptus sp.; e mata ciliar, devido à sua forma estreita e à área pequena.

De posse das curvas de nível (IBGE, 1979; 1981; 1984; escala 1:50000), foi gerado o Modelo Digital do Terreno (MDT) para obtenção do mapa de declividade no programa ArcGIS 9.2. Os mapas de declividade e hidrografia (IBGE, 1971; 1973; 1981, escala 1:50000) foram utilizados para geração do mapa de APP do município, considerando-se a distância de $30 \mathrm{~m}$ em torno de rios, visto que os rios do município possuem largura menor que 10 m, 30 m em torno de lagos e lagoas, $50 \mathrm{~m}$ em torno de nascentes e encostas com declividade acima de $45^{\circ}$, conforme estabelecido pelo artigo $4^{\circ}$ da Lei $\mathrm{n}^{\circ}$ 12.651, de 25 de maio de 2012, e no plano diretor do Município de Sorocaba (SOROCABA, 2007).

Realizou-se a sobreposição do mapa de fragmentos de vegetação natural e de APP para obtenção do mapa de APP preservadas e sem cobertura vegetal. Para testar o efeito da restauração das APP na estrutura da paisagem da área de estudo, compararam-se dois cenários: (1) cenário real (fragmentos de vegetação natural mapeados sobre fotografias aéreas de 2006) e (2) cenário ideal (fragmentos de vegetação natural de 2006 acrescidos da restauração das APP). No cenário ideal (com APP restauradas), evitou-se a utilização de regiões inviáveis para restauração como estradas (foi traçado um buffer de $50 \mathrm{~m}$ para todas as rodovias e excluídas as áreas).

Para ambos os cenários, foram calculadas as métricas estruturais da paisagem com relação à área, forma e conectividade dos fragmentos, usando a extensão VLATE 1.1. (Vector-Based Landscape Analysis Tools) do programa ArcGIS 9.2. O índice de forma foi calculado com a métrica (SHAPE), obtido pelo seguinte cálculo: $\mathrm{P} / \sqrt{\mathrm{A} / \mathrm{c}}$, sendo $\mathrm{P}=$ perímetro do fragmento, $\mathrm{A}=$ área de fragmento e c $=$ constante. Para o cálculo de conectividade, foi utilizada a métrica PROX, na qual foi calculada a área de todos os fragmentos dentro de um raio centrado no fragmento-alvo, dividida pelas distâncias desse fragmento elevadas ao quadrado, como mostrado na fórmula $\Sigma \mathrm{A} /(\Sigma \mathrm{D})^{2}$, sendo $\mathrm{A}=$ área dos fragmentos dentro do buffer e D = distância dos fragmentos dentro do buffer até o fragmento-alvo. Neste estudo, utilizou-se um raio de $100 \mathrm{~m}$ para simular o deslocamento de aves e pequenos mamíferos de Mata Atlântica (BOSCOLO; METZGER, 2009; FOREROMEDINA; VIEIRA, 2009). O programa R 2.7.1 foi utilizado no processamento dos dados.

\section{RESULTADOS}

O Município de Sorocaba possui uma área total de 4.5007,85 ha e apresentou 7.509,02 ha de cobertura florestal total no cenário real, o que representa 16,68\% do território municipal distribuído em 2.771 fragmentos (Figura 2). As APP preservadas constituem 50,60\%

Revista Árvore, Viçosa-MG, v.38, n.2, p.309-317, 2014 
dos remanescentes de vegetação natural da área de estudo, principalmente relacionadas às margens de cursos de água (Figura 2). Desse total, 1.715 (61,9\%) fragmentos apresentam área de até 1 ha, representando $7,29 \%$ da área total de cobertura de vegetação natural (Figura 3). Observa-se, nessa figura, que a grande maioria dos fragmentos $(94,84 \%)$ possuía áreas pequenas (menores que 10 ha) (Figura 3).

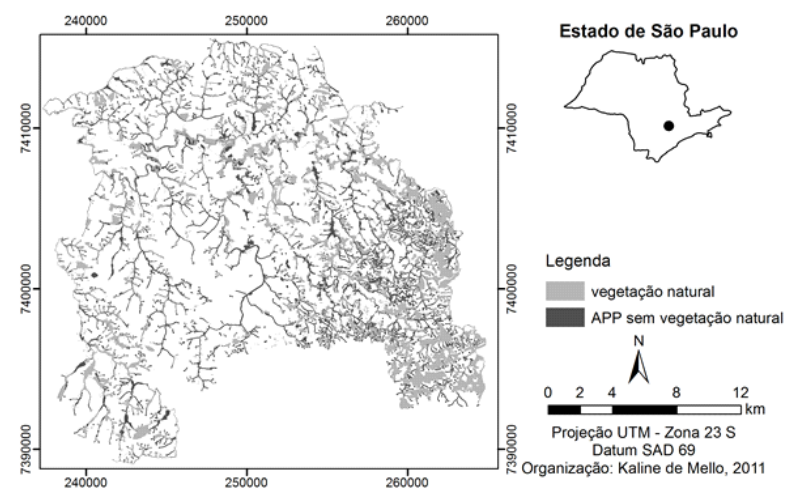

Figura 2 - Fragmentos de vegetação natural e APP sem cobertura florestal do Município de Sorocaba, Estado de São Paulo, Brasil.

Figure 2 - Fragments of native vegetation and Permanent Preservation Area without forest cover in the city of Sorocaba, São Paulo State, Brazil.

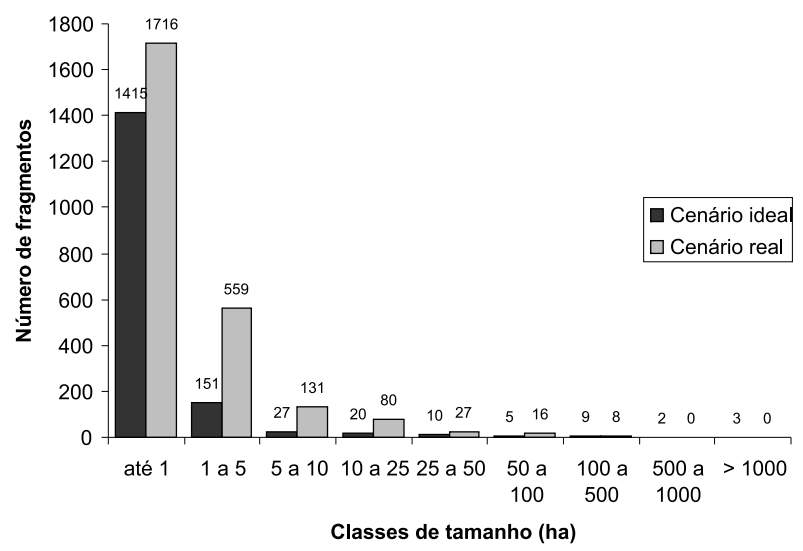

Figura 3 - Número de fragmentos de vegetação natural por classes de tamanho dos cenários real e ideal, Sorocaba, Estado de São Paulo, Brasil.

Figure 3 - Numbers of fragments of natural vegetation by size classes for real and ideal sceneries, Sorocaba, São Paulo State, Brazil.

Revista Árvore, Viçosa-MG, v.38, n.2, p.309-317, 2014
Utilizando a hidrografia e a declividade, obteve-se uma área total de APP para o município de 8.499 ha (18,9\% da sua área total). Da área legalmente protegida, 3.350 (44,7\%) possuem cobertura florestal no cenário real considerado, e o restante encontra-se sob a influência de áreas antropizadas (pasto, atividades agrícolas ou ocupação urbana) (Figura 2). Para a adequação total das áreas de APP a serem recuperadas, seria necessária a restauração de cerca de 3.800 ha (55,3\% das APP). Essa restauração representaria o acréscimo de 11,43\% de áreas naturais para Sorocaba, ou seja, o município passaria de $16,68 \%$ para $28,11 \%$ de cobertura florestal.

As métricas calculadas para os dois cenários (real e ideal) revelaram que, além do aumento de área total, houve redução no número de manchas de vegetação natural para o cenário ideal, passando de 2.537 para 1.642 fragmentos, e o surgimento de fragmentos extensos, o maior chegando a 3.400 ha, seguido de outros dois contendo 2.522 ha e 1.645 ha, enquanto no cenário real o maior apresentou apenas 314 ha (Figura 3). Ainda, houve aumento nos valores de conectividade, principalmente com relação aos fragmentos menores (Figura 4). No cenário real, 317 fragmentos apresentaram valores nulos ou próximos de zero de conexão e no cenário ideal, 215 fragmentos. Em ambos os cenários,

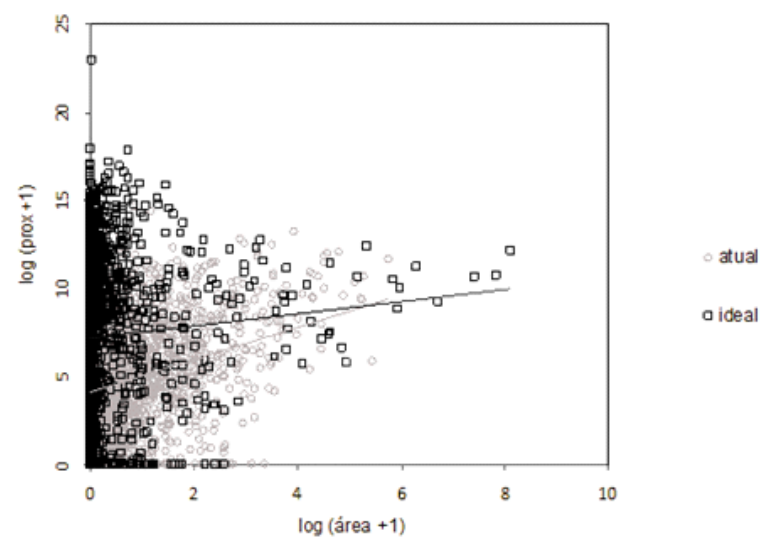

Figura 4 - Dispersão dos fragmentos de vegetação natural em função dos valores de área e conectividade (PROX) para os cenários real e ideal com as respectivas linhas de tendência, Sorocaba, Estado de São Paulo, Brasil.

Figure 4-Dispersion of the fragments with natural vegetation as a function of connectivity and area values (PROX) for real and ideal sceneries with their respective trend lines, Sorocaba, São Paulo State, Brazil. 
houve tendência de os maiores fragmentos apresentarem formas mais complexas e de os fragmentos menores possuírem formas mais arredondadas (Figura 5).

\section{DISCUSSÃO}

A cobertura de vegetação natural do município de Sorocaba $(16,68 \%)$ apresentou valor próximo ao de outros municípios do interior do Estado de São Paulo, que possuem formações de Cerrado e Floresta Estacional Semidecidual como Santa Cruz da Conceição (17,98\%) (FUSHITA, 2006), Araraquara (10,59\%) (MOSCHINI, 2005) e São Carlos (14,1\%) (CINTRA et al., 2004). Esses dados indicaram o quadro preocupante de conservação dessas formações vegetais e, consequentemente, das espécies ameaçadas desses hábitats, como é o caso de Copaifera langsdorffii e Machaerium villosum, que ocorrem somente na Floresta Estacional Semidecidual, dentro do bioma Mata Atlântica, e são encontradas no Município de Sorocaba (COELHO, 2013).

Os resultados evidenciaram que a cobertura florestal remanescente do município de Sorocaba está essencialmente associada à presença de corpos hídricos e ao relevo mais acentuado, ou seja, áreas de difícil acesso à ocupação humana, padrão comum para a

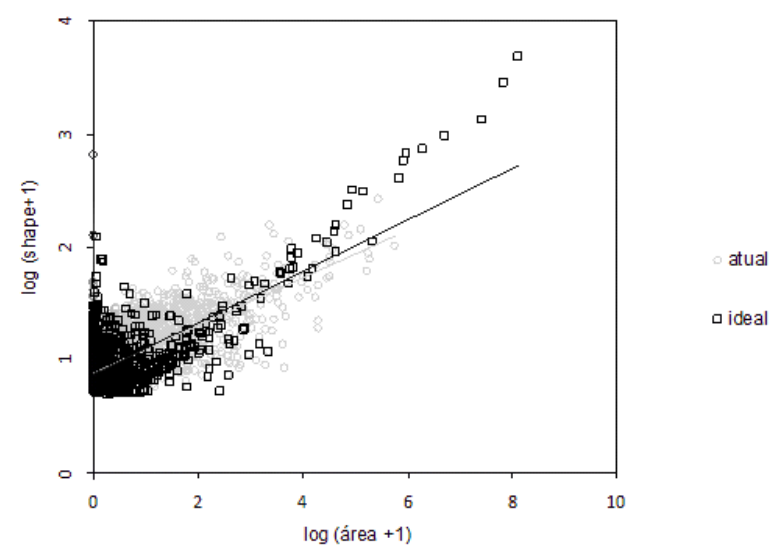

Figura 5 - Dispersão dos fragmentos de vegetação natural em função dos valores de área e forma (SHAPE) para os cenários real e ideal com as respectivas linhas de tendência, Sorocaba, Estado de São Paulo, Brasil.

Figure 5-Dispersion of the fragments with natural vegetation as a function of shape and area values (SHAPE) for real and ideal sceneries with their respective trend lines, São Paulo State, Brazil. distribuição dos remanescentes de Mata Atlântica (SILVA et al., 2007) e de outros biomas ameaçados no mundo (VELDKAMP; LAMBIN, 2001; DITT et al., 2008; ROMPRÉ et al., 2008). Topografia complexa é frequentemente associada com florestas mais maduras do que as áreas com terreno mais plano, pois o acesso à colheita e ao desenvolvimento é limitado (VELDKAMP; LAMBIN, 2001; SILVAet al., 2007; DITT, 2008; ROMPRÉ et al., 2008).

Estudo recente sobre a composição florística de um trecho de vegetação natural em zona ripária no município de Sorocaba apresentou seis espécies ameaçadas de extinção, 11 espécies endêmicas da Mata Atlântica e seis espécies restritas à Floresta Estacional Semidecidual, evidenciando a importância das APP do município para a conservação de espécies importantes da flora nativa (COELHO, 2013), uma vez que nosso estudo apontou que metade da cobertura florestal remanescente de Sorocaba está presente em APP (50,60\%).

Outros estudos analisaram a importância dos corredores de mata ciliar como hábitat para a fauna em paisagens fragmentadas de florestas tropicais (LEES; PERES, 2008; NAXARA, 2008), mostrando que os corredores florestados podem tanto facilitar a movimentação de indivíduos de determinadas espécies entre as manchas de hábitat disponíveis como funcionar como hábitat, abrigando populações residentes que conectam as populações das manchas remanescentes na paisagem (CHETKIEWICZ et al., 2006; LUSSIER et al., 2006; CASTÉLON; SIEVING, 2007). Assim, a manutenção, implantação e restauração de elementos lineares conectando fragmentos podem ser utilizadas como estratégias de conservação, pois potencialmente amenizariam os efeitos negativos da fragmentação sobre abiodiversidade(SAUNDERS; HOBBS, 1991; DOWNES et al., 1997; NAXARA, 2008). Além da importância para a conservação da biodiversidade em paisagens fragmentadas, a restauração das APP contribuiria para a diminuição de impactos ambientais relacionados principalmente à qualidade da água, como assoreamento dos corpos de água, retenção de poluentes e processos erosivos, gerando benefícios tanto para a qualidade de vida humana quanto para a sobrevivência de espécies aquáticas (TERESA; CASATTI, 2010).

O novo Código Florestal (BRASIL, 2012) estabelece algumas especificações para a recomposição de vegetação das APP em áreas consolidadas, que podem

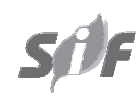

Revista Árvore, Viçosa-MG, v.38, n.2, p.309-317, 2014 
chegar a 5 m em relação à margem dos rios, conforme artigo 61-A. Essa medida pode ter impacto para a biodiversidade, visto que corredores muito estreitos perdem parte de sua utilidade por favorecerem unicamente espécies generalistas, as quais suportam os efeitos de borda (METZGER, 2010). Estudos feitos com diferentes grupos taxonômicos indicam a necessidade de uma faixa mínima de $100 \mathrm{~m}$ de área florestada para a persistência das espécies (TUBELIS et al., 2004; LEES; PERES, 2008), mostrando que a extensão das APP deveria ser expandida e não reduzida (METZGER, 2010). Para mamíferos e aves, especificamente, um dos efeitos principais da redução das APP seria o aumento do efeito de borda, o qual afetaria diretamente a produção de sementes e frutos das árvores e, consequentemente, a disponibilidade de alimento para ambos os grupos, além da redução de hábitat para manutenção de quantidades mínimas de indivíduos na paisagem (GALETTI et al., 2010).

A análise do cenário ideal evidenciou que a restauração das APP podem tanto aumentar a área total de cobertura de vegetação do município quanto significativamente o tamanho das manchas de hábitat, com o surgimento de fragmentos com quase 10 vezes o tamanho dos fragmentos do cenário real, ultrapassando 3.000 ha. O incremento no tamanho dos fragmentos está diretamente relacionado à diminuição do número de fragmentos quase pela metade, devido à junção entre eles, formando corredores ripários extensos.

Os valores de forma corroboram essa afirmação, uma vez que a tendência observada tanto no cenário real quanto no cenário ideal indica que os fragmentos maiores possuem formas mais alongadas e complexas, possivelmente por estarem conectados por corredores de vegetação ciliar, como encontrado por Ribeiro et al. (2005) e como ocorre em outros municípios do interior de São Paulo (FUSHITA, 2006; GREGGIO et al., 2009) e em grande parte da distribuição de Mata Atlântica no Brasil (SILVA, 2007). Por ser um município com alta taxa de urbanização, com 99\% das pessoas residentes na área urbana (IBGE, 2010b), as manchas de vegetação de Sorocaba tendem a permanecer nas várzeas, fundo de vale e áreas alagáveis, onde a ocupação humana é dificultada ou há problemas com enchentes.

$\mathrm{O}$ atual quadro de escassez de remanescentes florestais no município de Sorocaba gera uma demanda por ações imediatas de conservação e restauração ecológica. Uma vez que os remanescentes florestais se apresentam, em grande parte, com tamanhos pequenos ou muito alongados, além do cumprimento do código florestal, o município deve implantar ações com o intuito de aumentar as áreas florestais e conservar fragmentos que potencialmente abriguem maior número de espécies, como a criação de Unidades de Conservação de Proteção Integral e programas de reflorestamento, priorizando áreas de conexão entre fragmentos.

A expansão urbana do município deve ser planejada conjuntamente com a conservação das áreas naturais, integrando os instrumentos legais vinculados aos aspectos ambientais, sociais e econômicos, visando ao estabelecimento de cidades mais sustentáveis.

\section{CONCLUSÃO}

Os remanescentes florestais em Sorocaba apresentaram-se intimamente ligados à presença de corpos de água e terrenos com maior declividade, ou seja, nas APPs, onde a ocupação urbana foi dificultada e existe o dispositivo legal de proteção. As APPs conservadas representam cerca de 50\% dos remanescentes do município.

A restauração das APP aumentaria tanto a área total de cobertura florestal do município quanto, significativamente, o tamanho dos fragmentos e a conexão entre eles, formando extensos corredores de mata ripária. Dessa maneira, o simples cumprimento da legislação atuaria positivamente na conservação da biodiversidade em um município urbano, como é o caso de Sorocaba, especialmente na conservação de espécies de fauna que dependem de ambientes florestados e não conseguem sobreviver em áreas muito pequenas ou transpor áreas abertas. Entretanto, as alterações no novo Código Florestal representam um retrocesso na conservação da natureza no país, à medida que torna cada vez mais permissivo o desmatamento de áreas sensíveis e ecologicamente importantes. Dessa forma, outra estratégia importante para manutenção da vegetação natural nessas áreas seria a criação de Unidades de Conservação de Proteção Integral, cujas áreas deveriam ser maiores que os limites legais de proteção do Código Florestal.

\section{REFERÊNCIAS}

ALBERTI, M. Maintaining ecological integrity and sustaining ecosystem function in urban areas.

Current Opinion in Environmental Sustainability, v.2, n.3, p.178-184, 2010. 
BANKS-LEITE, C. et al. Comparing species and measures of landscape structure as indicators of conservation importance. Journal of Applied Ecology, v.48, n.3, p.706-714, 2011.

BATEMAN, P. W.; FLEMING, P. A. Big city life: carnivores in urban environments. Journal of Zoology, v.287, n.1, p.1-23, 2012.

BOSCOLO, D.; METZGER, J. P. Is bird incidence in Atlantic forest fragments influenced by landscape patterns at multiple scales?

Landscape Ecology, v.24, p.907-918, 2009.

BRASIL. Lei $n^{0}$ 12.651, de 25 de maio de 2012. Disponível em: <http://

www.planalto.gov.br/ccivil_03/_Ato2011-2014/ 2012/Lei/L12651.htm> Acesso em: 25 nov. de 2012.

CASTÉLON, T. D.; SIEVING, K. E. An experimental testo f matrix permeability and corridor use by na endemic understory bird. Conservation

Biology, v.20, n.1, p.135-145, 2007.

CHETKIEWICZ, C. L. B.; SAINT CLAIR, C. C.; BOYCE, M. S. Corridors for conservation: integrating pattern and process. Annual Review of Ecology, Evolution and Systematics, v.37, p.317-342, 2006.

CINTRA, R. H. et al. Análise qualitativa e quantitativa de danos ambientais com base na instauração e registros de instrumentos jurídicos. In: SANTOS, J. E. et. al. Faces da Polissemia da Paisagem: ecologia, planejamento e percepção. São Carlos: RiMa, 2004.

\section{COELHO, S. Estudo da vegetação do} Parque Natural Municipal "Corredores da Biodiversidade" (PNMCBio), Sorocaba/SP. Dissertação (Mestrado em Susentabilidade na Gestão Ambiental) - Universidade Federal de São Carlos, Campus de Sorocaba, 2013.

CONGAlTON, R. G.; GREEN, K. Assessing the accuracy of remotely sensed data: principles and practices. New York: Lewis Publishers, 1998. p.60.

DITT, E. H. et al. Defying legal protection of Atlantic Forest in the transforming landscape around the Atibainha reservoir, South-Eastern Brazil. Landscape and Urban Planning, v.86, p.276-283, 2008.
DOWNES, S. J.; HANDASYDE, K. A.; ELGAR, M. A. Variation in the use of corridors by introduced and native rodents in south-eastern Australia.

Biological Conservation, v.82, p.379-383, 1997.

FORERO-MEDINA, G.; VIEIRA, M. V. Perception of a fragmented landscape by Neotropical marsupials: effects of body mass and environmental variables. Journal of Tropical Ecology, v.25, n.1, p.53-62, 2009.

FUSHITA, A. T. Análise da fragmentação de áreas de vegetação natural e seminatural do município de Santa Cruz da Conceição, São Paulo, Brasil. 2006. 125f. Dissertação (Mestrado em Ecologia e Recursos Naturais) - Universidade Federal de São Carlos, São Carlos, 2006.

GALETTI, M. et al. Mudanças no Código Florestal e seu impacto na ecologia e diversidade de mamíferos no Brasil. Biota Neotropica, v.10, n.4, p.47-52, 2010.

GOMES, V.; RIBEIRO, R.; CARRETERO, M. A. Effects of urban habitat fragmentation on common small mammals: species versus communities.

Biodiversity and Conservation, v.20, n.14, p.3577-3590, 2011.

GREGGIO, T. C.; PISSARRA, T. C. T.; RODRIGUES, F. M. Avaliação dos fragmentos florestais do município de Jaboticabal-SP.

Revista Árvore, v.33, n.1, p.117-124, 2009.

GRIMM, N. B. et al. The changing landscape: ecosystem responses to urbanization and pollution across climatic and societal gradients. Frontiers in Ecology and the Environment, v.6, n.5, p.264-272, 2008.

GRIMM, N. B. et al. Integrated approaches to long-term studies of urban ecological systems. Bioscience, v.50, n.7, p.571-584, 2000.

INSTITUTO BRASIELIRO DE GEOGRAFIA E ESTATISTICA - IBGE. Atlas Nacional do Brasil Milton Santos. Brasília: 2010a.

INSTITUTO BRASIELIRO DE GEOGRAFIA E ESTATISTICA - IBGE. Cidades. São Paulo/ Sorocaba, 2010b. Disponível em: <http:// www.ibge.gov.br/cidadesat/topwindow.htm?!>. Acesso em: 22 out. 2012.

Revista Árvore, Viçosa-MG, v.38, n.2, p.309-317, 2014 
INSTITUTO BRASIELIRO DE GEOGRAFIA E ESTATISTICA - IBGE. Região Sudeste do Brasil. 1:50.000. Folha SF-23-Y-C-I-4/ MI-2765/4: Boituva: 1979.

INSTITUTO BRASIELIRO DE GEOGRAFIA E ESTATISTICA - IBGE. Região Sudeste do Brasil. 1:50.000. Folha SF-23-Y-C-II-3/ MI-2766/3: Itu: 1981.

INSTITUTO BRASIELIRO DE GEOGRAFIA E ESTATISTICA - IBGE. Região Sudeste do Brasil. 1:50.000. Folha SF-23-Y-C-V-1/ MI-2792/1: Sorocaba: 1981.

INSTITUTO BRASIELIRO DE GEOGRAFIA E ESTATISTICA - IBGE. Região Sudeste do Brasil. 1:50.000. Folha SF-23-Y-C-IV2/MI-2791/2: Salto de Pirapora: 1984.

KAYE, J. P. et al. A distinct urban biogeochemistry? Trends in Ecology \& Evolution, v.21, n.4, p.192-199, 2006.

KRONKA, F. J. N. (Ed). Inventário florestal da vegetação natural do Estado de São Paulo. São Paulo: Secretaria do Meio Ambiente/ Instituto Florestal/Imprensa Oficial, 2005.

LEES, A. C.; PERES, C. A. Conservation value of remnant riparian forest corridors of varying quality for amazonian birds and mammals. Conservation Biology, v.22, n.2, p.439-449, 2008.

LINDENMAYER, D. et al. A checklist for ecological management of landscapes for conservation. Ecology Letters, v.11, n.1, p.78-91, 2008.

LUSSIER, S. M. et al. Effects of habitat disturbance from residential development on breeding bird communities in riparian corridor. Environmental Management, v.38, n.3, p.504-521, 2006.

MCKINNEY, M. L. Urbanization, biodiversity, and conservation. Bioscience, v.52, n.10, 2002.

METZGER, J. P. Does the forest code has a scientific base? Natureza \& Conservacao, v.8, n.1, p.92-99, 2010.

Revista Árvore, Viçosa-MG, v.38, n.2, p.309-317, 2014
MOSCHINI, L. E. Diagnóstico e riscos ambientais relacionados à fragmentação de áreas naturais e semi-naturais da paisagem: estudo de caso, município de Araraquara, SP. 2005. 88f. Dissertação (Mestrado em Ecologia e Recursos Naturais) - Universidade Federal de São Carlos, São Carlos, 2005.

MOREIRA, M. A. Fundamentos do sensoriamento remoto e metodologia de aplicação. São José dos Campos: Instituto de Pesquisas Espaciais, 2001. p.422.

NAXARA, L. R. C. Importância dos corredores ripários para a fauna: pequenos mamíferos em manchas de floresta, matriz do entorno e elementos lineares em uma paisagem fragmentada de Mata Atlântica. 2008. 72f. Dissertação (Mestrado em Ciências) Universidade de São Paulo, São Paulo, 2008.

OLIVER, A. J. et al. Avifauna richness enhanced in large, isolated urban parks. Landscape and Urban Planning, v.102, n.4, p.215-225, 2011.

PICKETT, S. T. A. et al. Urban ecological systems: Linking terrestrial ecological, physical, and socioeconomic components of metropolitan areas. Annual Review of Ecology and Systematics, v.32, p.127-157, 2001.

PONÇANO, W. L. et al. Mapa geomorfológico do Estado de São Paulo. 1:1.000.000. São Paulo: IPT, 1981.

REIS, E.; LOPEZ-IBORRA, G. M.; PINHEIRO, R. T. Changes in bird species richness through different levels of urbanization: Implications for biodiversity conservation and garden design in Central Brazil. Landscape and Urban Planning, v.107, n.1, p.31-42, 2012.

RIBEIRO, C. A. A. S. et al. Desafio da delimitação de áreas de preservação permanente. Revista Árvore, v.29, n.2, p.203-212, 2005.

RIBEIRO, C. R. et al. The Brazilian Atlantic Forest: how much is left, and how is the remaining forest distributed? Implications for conservation.

Biological Conservation, v.142, p.11411153, 2009. 
ROMPRÉ, G.; ROBINSON, W. D.; DESROCHERS, A. Causes of habitat loss in a Neotropical landscape: The Panama Canal corridor. Landscape and Urban Planning, v.87, n.2, p.129-139, 2008.

ROSNER, B. Fundamentals of Biostatistics. Boston: Duxbury Press, 2006. 876p.

\section{SAUNDERS, D. A.; HOBBS, R. J. Nature} conservation 2: the role of corridors. New South Wales: Surrey Beatty \& sons/Chipping Norton, 1991. p.456.

SCHINDLER, S. et al. Multiscale performance of landscape metrics as indicators of species richness of plants, insects and vertebrates. Ecological Indicators, v.31, p.41-48, 2012.

SCOLOZZI, R.; GENELETTI, D. A multi-scale qualitative approach to assess the impact of urbanization on natural habitats and their connectivity. Environmental Impact Assessment Review, v.36, p.9-22, 2012.

SILVA, W. G. S. et al. Relief influence on the spatial distribution of the Atlantic Forest cover at the Ibiúna Plateau, SP. Brazilian Journal of Biology, v.67, n.3, p.403-411, 2007.

SOROCABA (Município). Prefeitura Municipal. Lei ${ }^{\circ}$ 8.181, de 05 de junho de 2007. Revisão da Lei 7.122 de 04/6/2004, que instituiu o novo Plano Diretor de Desenvolvimento Físico Territorial do Município de Sorocaba, e dá outras providências. Sorocaba: 2007.

SOROCABA (Município). Prefeitura Municipal Coordenadoria Municipal de Defesa Civil.

Levantamento de áreas de risco de Sorocaba. Sorocaba: 2010.
STEINER, F. Landscape ecological urbanism: Origins and trajectories. Landscape and Urban Planning, v.100, n.4, p.333-337, 2011.

TERESA, F. B.; CASATTI, L. Importância da vegetação ripária em região intensamente desmatada no sudeste do Brasil: um estudo com peixes de riacho. Pan-American Journal of Aquatic Sciences, v.5, n.3, p.44-453, 2010.

THRELFALL, C. G.; LAW, B.; BANKS, P. B. Influence of Landscape Structure and Human Modifications on Insect Biomass and Bat Foraging Activity in an Urban Landscape. Plos One, v.7, n.6, p.10, 2012.

TUBELIS, D. P.; COWLING, A.; DONNELLY, C. Landscape supplementation in adjacent savannas and its implications for the design of corridors for forest birds in the central Cerrado, Brazil. Biological Conservation, v.118, n.3, p.353-364, 2004.

UN. World Urbanization Prospects: The 2009 Revision. New York: 2010. 47p.

VELDKAMP, A.; LAMBIN, E. F. Predicting landuse change. Agriculture Ecosystems and Environnent, v.85, n.1, p.1-6, 2001.

YORK, A. M. et al. Land fragmentation under rapid urbanization: A cross-site analysis of Southwestern cities. Urban Ecosystems, v.14, n.3, p.429-455, 2011.

ZHOU, Y. et al. Urban green space planning based on computational fluid dynamics model and landscape ecology principle: A case study of Liaoyang City, Northeast China. Chinese Geographical Science, v.21, n.4, p.465-475, 2011. 
